\title{
Immunologic Studies in von Willebrand's Disease
}

\author{
EVIDENCE THAT THE ANTIHEMOPHILIC FACTOR (AHF) \\ PRODUCED AFTER TRANSFUSIONS LACKS AN ANTIGEN \\ ASSOCIATED WITH NORMAL AHF AND THE \\ INACTIVE MATERIAL PRODUCED BY PATIENTS \\ WITH CLASSIC HEMOPHILIA
}

\author{
Bruce Bennett, Oscar D. Ratnoff, and Jack Levin \\ From the Department of Medicine, Case Western Reserve University School of \\ Medicine and University Hospitals of Cleveland, Cleveland, Ohio 44106 and \\ the Johns Hopkins University School of Medicine and Hospital, \\ Baltimore, Maryland 21205
}

\begin{abstract}
A в S T R A C T Antihemophilic globulin (AHF, factor VIII) levels were measured by a standard coagulation assay and by an immunological technique before and serially after infusion of fresh frozen plasma or cryoprecipitate into patients with von Willebrand's disease. Initial levels of AHF, measured both as procoagulant and as antigen, were low. Immediately after transfusions, the rise in levels of AHF-like antigen was compatible with the quantity of antigen present in the infused plasma or cryoprecipitate. Thereafter, levels of antigen declined rapidly and reached preinfusion values in approximately $24 \mathrm{hr}$. In contrast, procoagulant activity remained elevated, and sometimes continued to rise, for longer periods of time. One possible explanation of this finding is that the AHF molecule produced by patients with von Willebrand's disease, in response to transfusion of as yet unidentified factors, lacks the antigenic site associated with the normal AHF molecule or the inactive molecule produced by patients with hemophilia A.
\end{abstract}

A preliminary report of these studies was presented at the Meetings of the American Society of Hematology, San Francisco, Calif., December 1971 (1).

Dr. Bennett is a Research Fellow of the Pink Family Foundation. Dr. Ratnoff is a Career Investigator of the American Heart Association. Dr. Levin is a John and Mary Markle Scholar in Academic Medicine and a recipient of a Research Career Development Award (5 KO4 HE 29906-3).

Received for publication 10 April 1972 and in reised form 13 June 1972.

\section{INTRODUCTION}

von Willebrand's disease is a familial hemorrhagic disorder characterized by an autosomal dominant mode of inheritance (2), a prolonged bleeding time (2), reduced levels of plasma antihemophilic factor. (AHF, factor VIII) ${ }^{1}(3)$, and decreased platelet retention in glass bead columns (4). It has been established recently, by means of a heterologous precipitating antibody to purified normal AHF, that the plasma of patients with classic sex-linked hemophilia A contains normal or increased concentrations of a molecule which precipitates with this antibody $(5,6)$. The half-life of AHF-like antigen after infusion into hemophilic subjects is significantly longer than that of AHF procoagulant (i.e., clot-promoting) activity suggesting that utilization of the antigenic material is in some way delayed in these patients (7). The plasma of patients with von Willebrand's disease contains reduced quantities of antigen (6). Infusion of plasma or plasma concentrates results in a delayed rise in the AHF clot-promoting activity in the blood of patients with von Willebrand's disease $(3,8)$. The magnitude and duration of this response is compatible with the production, in vivo, of functionally active AHF. We report here studies of changes in the levels of AHF clotpromoting activity and AHF-like antigen after infusions of fresh frozen plasma or cryoprecipitate into patients with von Willebrand's disease. Unexpectedly, the delayed

\footnotetext{
${ }^{1}$ Abbreviation used in this paper: AHF, antihemophilic factor.
} 
rise in levels of functional AHF clot-promoting agent was not accompanied by a rise in immunologically detectable AHF.

\section{METHODS}

The methods for assay of AHF clot-promoting activity, for purification of AHF from normal people, preparation of an antiserum to this, and the characteristics of the antiserum have been described in the accompanying paper (7) and previously (6). The method for the quantitation of AHF-like antigen employed in this study employed the application of concentrates of plasma to the wells of the antibody-containing agarose slides (6): this was necessary because the patients here studied had initial levels of AHF-like antigen too low to be detected by application of unconcentrated plasma to the wells.

Collection of blood samples and preparation of plasma was as previously described (6) with the exception that blood from the patients treated with fresh frozen plasma was mixed with one-ninth volume of $3.8 \%$ sodium citrate. The difference in the anticoagulants reflects the different choice of anticoagulant in our respective laboratories.

$1 \mathrm{U}$ of AHF clot-promoting activity is that amount present in $1 \mathrm{ml}$ of pooled whole normal plasma (6). $1 \mathrm{U}$ of AHFlike antigen is also the amount present in $1 \mathrm{ml}$ pooled whole normal plasma (6). It must be emphasized that the two techniques do not necessarily measure identical molecular entities, so the two unitages need not necessarily be interchangeable.

Patients with a diagnosis of von Willebrand's disease established on a basis of typical family and personal history of bleeding disorder, reduced levels of plasma AHF clotpromoting activity, and in most cases, a repeatedly prolonged bleeding time were hospitalized for treatment of a variety of bleeding episodes or, in one case for treatment of mammary carcinoma. All patients were female and all had received several transfusions of plasma or cryoprecipitate previously with the exception of the patient illustrated in Fig. 3 whose first hospital admission for bleeding was studied. Two patients received two infusions each of fresh frozen plasma and four received single infusions of cryoprecipitate.

\section{RESULTS}

Two patients received two infusions each of fresh frozen plasma. Fig. 1 illustrates the findings in one of these.

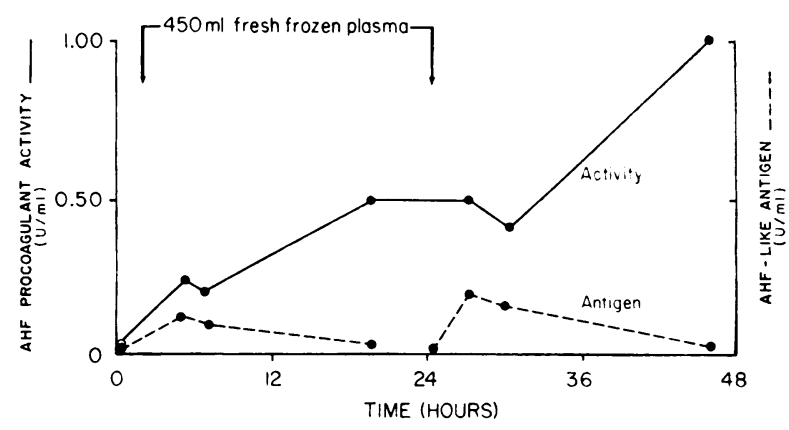

FIGURE 1 Changes in levels of AHF procoagulant activity (continuous line) and AHF-like antigen (interrupted line) after initial two infusions of fresh frozen plasma in a patient with von Willebrand's disease.

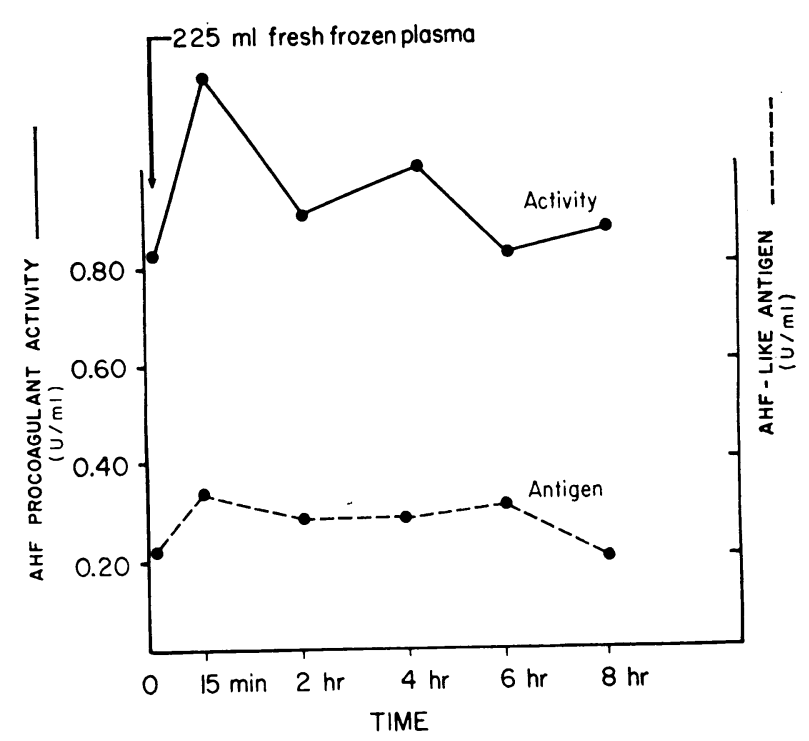

FIGURE 2 Levels of AHF procoagulant activity (continuous line) and AHF-like antigen (interrupted line) between two infusions of fresh frozen plasma in the same patient as Fig. 1 after she had been receiving $225 \mathrm{ml}$ fresh frozen plasma at 8 -hr intervals for 2 wk during a prolonged bleeding episode.

Peak levels of antigen, observed immediately after the infusions were low as was to be expected from the small amount of material administered. Thereafter, antigen levels declined rapidly and reached preinfusion values within $24 \mathrm{hr}$ on both occasions. In contrast, AHF clotpromoting activity continued to rise and the response to the two infusions was cumulative; a final level of 1.0 $\mathrm{U} / \mathrm{ml}$ plasma ( $100 \%$ of normal) was reached at a time when antigen level had returned to preinfusion values. Qualitatively similar results were observed in a second patient treated with fresh frozen plasma. Fig. 2 illustrates the observations made before and after infusion of 225 $\mathrm{ml}$ fresh frozen plasma into the patient illustrated in Fig. 1 at a time when she had received the same volume of fresh frozen plasma at intervals of $8 \mathrm{hr}$ for $2 \mathrm{wk}$ during a severe bleeding episode. At the time of these observations she was therefore responding to infusions of plasma given $8,16,24$, etc., hr previously and the observations indicate a sustained high level of AHF clotpromoting activity (approximately $1.0 \mathrm{U} / \mathrm{ml}$ ) with a much lower level of antigen (approximately $0.25 \mathrm{U} / \mathrm{ml}$ ). Infusion of the small amount of plasma resulted in small increases in both antigen and activity.

Fig. 3 presents the observations made in a patient with von Willebrand's disease who received a single infusion of cryoprecipitate. Very high levels of antigen were observed immediately after the infusion with a less marked rise in clot-promoting activity. As preparations of cryoprecipitate contain considerably higher levels of antigen than clot-promoting activity (7) this was an expected 


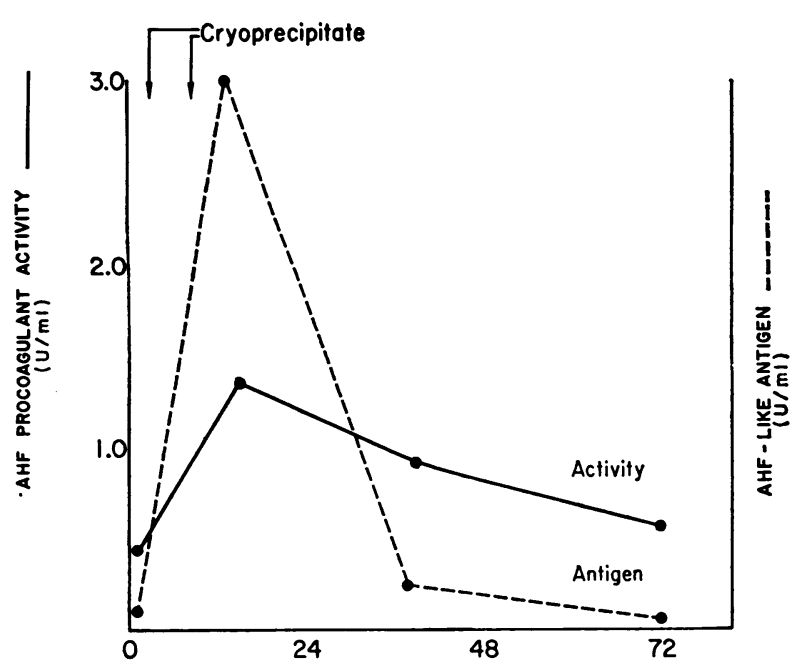

TIME (HOURS)

FIGURE 3 Changes in levels of AHF procoagulant activity (continuous line) and AHF-like antigen (interrupted line) after infusion of cryoprecipitate. The patient was a 6 yr old child (weight $20 \mathrm{~kg}$ ) who sustained an injury to her eye with severe intraocular bleeding. As initial treatment, seven bags of cryoprecipitate were infused on each of the two occasions indicated by the arrows. (One bag of cryoprecipitate is derived from $225 \mathrm{ml}$ plasma).

finding. As in the cases treated with fresh frozen plasma, antigen levels declined rapidly to reach approximately preinfusion values in $24 \mathrm{hr}$, while the level of AHF clotpromoting activity remained significantly elevated between 48 and $72 \mathrm{hr}$. It should be pointed out that in this patient, in contrast to those treated with fresh frozen plasma, the infused material contained a large amount of AHF clot-promoting activity which is represented by the large increase evident in the first postinfusion observation; this immediate large rise tends to obscure the delayed rise in AHF clot-promoting activity typical of von Willebrand's disease which is represented here by the elevated levels noted at 40 and $72 \mathrm{hr}$. Results qualitatively similar to these were observed in three further patients treated with cryoprecipitate infusions.

\section{DISCUSSION}

Zimmerman, Ratnoff, and Powell (6) using quantitative immunoelectrophoresis, showed that patients with von Willebrand's disease had reduced levels of AHF-like antigen, while plasma of patients with classic hemophilia contained normal quantities of AHF-like antigen. Earlier studies, which employed a variety of antibody preparations, had produced less clear-cut results $(9-11)$, but several groups have now confirmed this finding (12-15).

Earlier studies from this laboratory, which showed correlation of levels of AHF antigen and clotting ac- tivity in normal people and, at reduced levels, in those with von Willebrand's disease (6), were carried out in unstressed subjects. Recent studies indicate that normal people stressed by strenuous exercise respond with a parallel rise in AHF clot-promoting activity and antigen $(16,17)$, as do some patients undergoing the stress of pneumoencephalography (17). Normal pregnancy is also associated with a parallel rise in levels of antigen and clotting activity (17). These observations, together with the finding that highly purified preparations of AHF from normal plasma possess both antigen and clot-promoting activity (6), suggest that the sites determining these activities are carried on the same or closely similar molecules, or that a relatively fixed proportion of the material detected as antigen in normal plasma has functional clot-promoting activity.

The divergence of levels of AHF clot-promoting activity and AHF-like antigen after transfusion of patients with von Willebrand's disease contrasts sharply with that observed in classic hemophilia (7), and necessitates a different consideration of their role in the AHF "economy" of patients with the disorder. The very rapid disappearance of the antigen from the blood of patients with von Willebrand's disease suggests that these people are capable of metabolizing or destroying antigen in a manner not available to hemophiliacs. This, together with the subsequent increase in AHF clotting activity might suggest that antigen is present on a precursor molecule which patients with von Willebrand's disease produce in reduced quantities but can convert to an agent with $\mathrm{AHF}$ clotting activity once it is supplied to them. The relationship of this material to the functional AHF of normal subjects is difficult to determine at present as the material produced after transfusion by von Willebrand's disease subjects is not associated with antigen, detectable with the antiserum we used, whereas in normal people AHF clotting activity and antigen appear closely associated as indicated by the observations detailed above.

Possible interpretations of the available data on $\mathrm{AHF}$ in normals, hemophiliacs and those with von Willebrand's disease may be summarized as follows:

(a) The antigen detected by the rabbit antiserum appears only on a precursor of the AHF clot-promoting agent. This is suggested by the observations reported here on von Willebrand's disease, and the observations on the effect of infusion of cryoprecipitate into hemophiliacs (7) are compatible with this concept. However there is at present no direct evidence that, in normal plasma, the AHF clot-promoting activity and the antigen are separable entities. The reverse is suggested by the fact that the antiserum to normal purified AHF destroys functional AHF clot-promoting activity, and studies in progress, but as yet incomplete, indicate that the antiserum to the nonfunctional material purified from hemophilic plasma 
similarly destroys the clot-promoting activity of normal $\mathrm{AHF}$; both these observations suggest that the antigenic site and that determining clot-promoting activity appear on the same molecule or on two species of molecule both of which are essential for the expression of AHF clotpromoting activity.

(b) The antiserum detects only the final functional form of AHF. This would necessitate that the agent present in the plasma of hemophiliacs be regarded as antigenically normal but functionally abnormal, perhaps because of an abnormal configuration or amino acid substitution at the active site. It is difficult to reconcile this concept with the fact that hemophilic plasma can simulate the appearance of functional AHF clot-promoting activity in plasma of patients with von Willebrand's disease. In a similar way this interpretation would regard the material produced after transfusion of patients with von Willebrand's disease as abnormal in that it lacks the antigen.

(c) The antigen is present on both the normal functional AHF clot-promoting agent and a precursor material. This would require that the material appearing after transfusion of patients with von Willebrand's disease be regarded as abnormal as it lacks the antigen, but is compatible with the other observations.

It is not possible at present to state which of the alternatives proposed (or omitted) is correct. Preliminary results of studies in progress, suggest that the agent produced by von Willebrand's disease patients after transfusion is as susceptible as is normal AHF clotting activity to inactivation by human circulating anticoagulants to $\mathrm{AHF}$, but it is considerably more resistant to inhibition by the rabbit antiserum. This serves to emphasize its functional similarity and antigenic dissimilarity to normal AHF. Further study in this area is in progress.

A further point of interest arises from our observations, which may have a bearing on the correction of the bleeding time after infusion of plasma or cryoprecipitate into patients with von Willebrand's disease. Bouma, van Mourik, Mochtar, and Sixma have demonstrated that purified AHF from normal subjects (which contains the antigen) and the material prepared in an identical manner from hemophilic plasma (devoid of clotting activity but also containing the antigen) will correct, in vitro, the defective adhesiveness of platelets from patients with von Willebrand's disease (18). It is established that infusions of normal plasma or serum or plasma from hemophilic subjects (all of which contain the antigen) will correct the defect in platelet adhesiveness and the bleeding time in patients with von Willebrand's disease, but this effect lasts for only a few hours $(3,8,19)$, whereas the patient shows increased or increasing levels of $\mathrm{AHF}$ clot-promoting activity for longer periods. Our observation that this clot-promoting activity is not associ- ated with AHF-like antigen and that infused antigen is cleared from the circulation in a period comparable with that for which the bleeding time and platelet adhesiveness are corrected, supports the suggestion that the antigenic material corrects the abnormality in platelet function in von Willebrand's disease (18), whether AHF clot-promoting activity is present or not.

Although the precise nature and the abnormalities of the molecules produced by patients with von Willebrand's disease and classic hemophilia are not yet clear, the data available suggest that von Willebrand's disease may be due to a defect or a deficiency of a single protein; this, when infused into patients, corrects the bleeding time by restoring adhesiveness to platelets while it remains in the circulation, and stimulates, in a manner as yet undefined, production of an AHF molecule which has functional activity but lacks an antigenic site normally associated with AHF.

\section{ACKNOWLEDGMENTS}

This study was supported in part by Research Grants HE 01601 and 01661 from the National Heart and Lung Institute, The National Institutes of Health, U. S. Public Health Service, and in part by grants from the American Heart Association.

\section{REFERENCES}

1. Bennett, B., J. Levin, and O. D. Ratnoff. 1971. Immunological properties of the antihemophilic material produced by patients with von Willebrand's disease. Blood. J. IIematol. 38: 817. (Abstr.)

2. von Willebrand, E. 1926. Hereditary pseudohemophilia; description; previously observed cases. Fin. Lakaresallsk. Handl. 68: 87.

3. Nilsson, I. M., M. Blombäck, and B. Blombäck. 1959. Von Willebrand's disease in Sweden. Its pathogenesis and treatment. Acta Med. Scand. 164: 263.

4. Salzmann, E. W. 1963. Measurement of platelet adhesiveness. A simple in vitro technique demonstrating an abnormality in von Willebrand's disease. J. Lab. Clin. Mcd. 62: 724 .

5. Zimmerman, T. S., and O. D. Ratnoff. 1970. Detection of carriers of classic hemophilia using an immunoassay for antihemophilic factor (Factor VIII). J. Lab. Clin. Mcd. 76: 983. (Abstr.)

6. Zimmerman, T. S., O. D. Ratnoff, and A. E. Powell. 1971. Immunologic differentiation of classic hemophilia (factor VIII deficiency) and von Willebrand's disease. With observations on combined deficiencies of antihemophilic factor and proaccelerin (factor $\mathrm{V}$ ) and on an acquired circulating anticoagulant against antihemophilic factor. J. Clin. Invest. 50: 244.

7. Bennett, B., and O. D. Ratnoff. 1972. Studies on the response of patients with classic hemophilia to transfusion with concentrates of antihemophilic factor. A difference in the half-life of antihemophilic factor as measured by procoagulant and immunologic techniques. J. Clin. Invest. $51: 2593$.

8. Cornu, P., M. J. Larrieu, J. Caen, and J. Bernard. 1963. Transfusion studies in von Willebrand's disease: effect 
on bleeding time and Factor VIII. Br. J. Haematol. 9: 189.

9. Uszynski, L. 1967. The immunological properties of Factor VIII. II. The immunoelectrophoretic studies. Thromb. Diath. Haemorrh. 18: 325.

10. Denson, K. W. E., R. Biggs, M. E. Haddon, R. Borrett, and $\mathrm{K}$. Cobb. 1969. Two types of haemophilia $\left(\mathrm{A}^{+}\right.$and $\left.\mathrm{A}^{-}\right)$: a study of 48 cases. Br. J. Haematol. 17: 163.

11. Goudemand, M., M. Foucaut, A. Hutin, and A. ParquetGernez. 1963. Les anticoagulants circulants anti-facteur VIII au cours de l'hemophilie A. (Nature et mécanisme d'action.) Nouv. Rev. Fr. Hematol. 3: 703.

12. Stites, D. P., E. J. Hershgold, J. D. Perlman, and H. H. Fudenberg. 1971. Factor VIII detection by hemagglutination inhibition: hemophilia A and von Willebrand's disease. Science (Wash. D. C.). 171: 196.

13. Bennett, E., and E. R. Huehns. 1970. Immunological differentiation of three types of haemophilia and identification of some female carriers. Lancet. 2: 956.

14. Shapiro, G. A., and P. A. McKee. 1970. Demonstration of a non-functional antihemophilic factor (Factor VIII) in classic hemophilia. Clin. Res. 18: 615. (Abstr.)
15. Hoyer, L. W. 1972. Immunologic studies of antihemophilic factor (AHF factor VIII). III. Comparative binding properties of human and rabbit anti-AHF. Blood J. Hematol. 39: 481.

16. Rizza, C. R., and J. Eipe. 1971. Exercise, Factor VIII and the spleen. Br. J. Haematol. 20: 629.

17. Bennett, B., and O. D. Ratnoff. 1972. Changes in antihemophilic factor (AHF) procoagulant activity and AHF-like antigen in normal pregnancy and following physical exercise and pneumoencephalography. J. Lab. Clin. Med. In press.

18. Bouma, B. N., Y. Wiegerinck, J. J. Sixma, J. A. van Mourik, and I. A. Mochtar. 1972. Immunochemical characterization of purified anti-haemophilic Factor A (Factor VIII) which corrects abnormal platelet retention in von Willebrand's disease. Nat. New Biol. 236: 104.

19. Salzman, E. W., and A. Britten. 1964. In vitro correction of defective platelet adhesiveness in von Willebrand's disease. Fed. Proc. 23: 239. (Abstr.)

20. Salzman, E. W. 1967. Measurement of platelet adhesiveness: progress report. Thromb. Diath. Haemorrh. Suppl. 26: 303 . 